\title{
Self-injurious behaviour in people with intellectual disability.
}

\author{
Oliver, C. and Richards, C. \\ Cerebra Centre for Neurodevelopmental Disorders, \\ School of Psychology, \\ University of Birmingham
}

Please use this reference when citing this work:

Oliver, C. and Richards, C. (2010). Self-injurious behaviour in people with intellectual disability. Current Opinion in Psychiatry, 23, 412-416. DOI:10.1097/YCO.0b013e32833cfb80

The Cerebra Centre for Neurodevelopmental Disorders,

School of Psychology, University of Birmingham, Edgbaston, Birmingham, B15 2TT Website: www.cndd.Bham.ac.uk E-mail: cndd-enquiries@contacts.bham.ac.uk 


\begin{abstract}
Purpose of review. To provide a critical evaluation of a broad range of peer reviewed published studies of relevance to self-injurious behaviour in people with intellectual disability and autism spectrum disorders.

Recent findings. The review covers the prevalence of self-injurious behaviour, the characteristics of those showing the behaviour, self-injury in autism spectrum disorders, biological influences on self-injurious behaviour, behavioural assessment and psychological and pharmacological interventions

Summary: The recent literature describes systematic evaluation of the efficacy of aripiprazole, and parent training combined with risperidone. Meta analyses of behavioural interventions provide evidence of their efficacy and related research describes beneficial modification to behavioural assessment procedures. The prevalence literature provides data on individual characteristics that are associated with persistence and presence of self-injury and that might be considered as risk markers. Pain behaviour appears to be associated with self-injury with implications for theories of the involvement of endorphins and as a casual factor. In combination these research findings demonstrate the multiple influences on selfinjurious behaviour that must be taken into account in the assessment, formulation, intervention process.
\end{abstract}

Keywords: Self-injurious behaviour; autism spectrum disorder; behavioural phenotypes; pharmacology; behavioural intervention 


\section{Introduction}

There are discernible trends in the recent research literature on self-injurious behaviour. One is the quality and strength of evidence for interventions with the emergence of medication trials of robust design and meta-analyses of behavioural procedures. The second is the identification of individual characteristics that are associated with self-injury becoming incorporated into causal models. Finally, there is some evidence of integration of biological and environmental influence in constructing interventions and the examination of likely contextual variables on intervention. These trends are positive and can ultimately influence the quality and availability of clinical services. The review is divided into sections on prevalence and individual characteristics, autism spectrum disorders, biological influences on self-injury, behavioural assessment and psychological and pharmacological intervention.

\section{Prevalence and individual characteristics}

Cooper et al. [1**] reported a prevalence rate of $4.9 \%$ from a rigorous longitudinal study of prevalence and remission rates of self-injurious behaviour in adults with intellectual disabilities. Interestingly, they report a remission rate of $38.2 \%$, higher than that found in previous studies, and attribute discrepancies in this finding to the use of inpatient samples in previous research. The authors also investigated risk markers for persistence of self-injury. In line with recently published research, they demonstrated that at a single time point, lower ability [2,3], Attention Deficit and Hyperactivity Disorder (ADHD), visual impairments, compromised communication $[4,5]$ and autism spectrum disorders $[2,6]$ were all associated with self-injurious behaviour. However, they found that only lower ability, ADHD and visual impairments were independently associated with self-injury over time. This important finding contributes to an understanding of how autism, ability and communication interact in their associations with self-injurious behaviour. Longitudinal studies of self-injurious behaviour should seek to replicate the findings of Cooper et al. [1**] and causal models should consider risk makers for onset, persistence and increasing severity of self-injurious behaviour.

The association between self-injurious behaviour and psychiatric disorders continues to receive significant research attention. When considering challenging behaviour more broadly, Felce and colleagues [7] reported a positive association between challenging behaviour scores and reaching threshold on a psychiatric screen. However, McCarthy et al. [8] demonstrated that there is no association between challenging behaviour and psychopathology, when gender, age and intellectual disability are controlled for. Interestingly, they report that only diagnosis of autism predicted challenging behaviour, not diagnosis of psychiatric conditions. Holden and Gitlesen [9**] presented data supporting a noteworthy interpretation: that the association between psychiatric symptoms and challenging behaviour may be due to overlap in the observed behaviours contributing to informants reporting psychopathology. More specifically, they demonstrated that $73 \%$ of the psychiatric symptoms reported by informants utilising the PAS-ADD, were based on observed challenging behaviour. However, with regard to self-injurious behaviour, this finding should be interpreted with caution as only $2.4 \%$ of the psychiatric symptoms were reported based solely upon the presence of self-injury. Nevertheless, this is a useful empirical demonstration of challenging behaviour being conceptualised by informants as a symptom of psychopathology, threatening the validity of any association between the two.

A relatively new stream of research has begun to consider self-injurious behaviour in individuals with mild/moderate intellectual disability [10,11]. This research should be 
commended for attempting to provide a synthesis of disparate fields of research across intellectual disabilities and typically developing individuals. Importantly, the authors suggest that approaches within mild/moderate populations must take into account cognitive as well as behavioural factors when assessing self-injury [10]. However, both studies take a predominantly qualitative approach, and therefore do not present empirical findings on prevalence, risk, causative factors or cognitive influences on self-injury. Consequently the utility of these studies in describing self-injury in this population is limited.

\section{Autism Spectrum Disorders}

Prevalence, risk markers, behavioural function and intervention for self-injurious behaviour has received much recent research attention within autism spectrum disorder (ASD) populations. Given the high reported prevalence of self-injurious behaviour in ASD investigating this behaviour in this population has importance. Matson, Boisjoli and Mahan [5] demonstrated that within an ASD population, self-injurious behaviour was associated with compromised receptive language and Murphy et al. [3] demonstrated that self-injurious behaviour was associated with severe intellectual disability in ASD. However, the diversity of ability and ASD phenomenology within these populations means that greater attention must be paid to accurately describing sample characteristics and confirming ASD diagnosis in order for any of the findings from the studies to be generalised.

Two recent studies focused on the behavioural function of self-injury in ASD. Love, Carr and Leblanc [12**] examined archival data from indirect, descriptive and experimental functional analyses conducted with 32 children with ASD and challenging behaviour. The authors reported that the majority of all challenging behaviour was maintained by positive social reinforcement and is therefore comparable to operant function in the wider developmental disabilities population. However, the findings of the study are not delineated by topography so it is not possible to draw conclusions about self-injurious behaviour specifically. O'Reilly and colleagues $\left[13^{*}\right]$ conducted experimental functional analyses with ten children with ASD who displayed challenging behaviour. This study takes an important step forward within the literature by confirming each child's diagnostic status with a psychiatrist or paediatrician and administering the Childhood Autism Rating Scale. The results of the functional analyses contrasts with the findings of Love et al. [12**] revealing that for eight of the ten children, challenging behaviour was maintained by automatic reinforcement. The authors suggest that this is evidence for differentiated behavioural function in ASD compared to developmental disabilities as a whole. However, as with the paper by Love et al. [12**] the study does not look at individual topographies of behaviour, but instead attempts to determine a single function for all challenging behaviour displayed by a child. Therefore, it is not possible to determine whether children with ASD have a different profile of behavioural function to their self-injurious behaviour. However, the heightened presence of automatic reinforcement may in part be supported by recent intervention research [14]. This demonstrated that a vibroacoustic music intervention produced a reduction in self-injurious behaviour, and that this reduction was greater in those with ASD than in those with developmental disabilities

Further intervention studies have also been reported. Both Soares et al. [15*] and Tiger, Fisher and Bouxsein $\left[16^{*}\right]$ conducted single case interventions with individuals with Asperger syndrome displaying self-injurious behaviour. Both interventions involved a selfmonitoring component, and demonstrated good efficacy in reducing self-injury. This novel 
approach should be incorporated in future research to determine its utility in broader intervention packages.

\section{Biological influences}

An important finding reported by May et al. [17**] identifies an association between a lowefficiency version of the MAOA gene and problem behaviour. This line of research warrants attention as it may, for example, offer a plausible account of within syndrome variability with regard to self-injury. A study by Symons et al. [18**] is of equal importance and identifies signs of pain in those showing chronic self-injury. The authors argue this might negate an elevated endorphin theory and may also indicate pain as a potential cause of self-injury.

Lesch-Nyhan syndrome is, arguably, the clearest example of a behavioural phenotype with severe self-injury prominent. Garcia et al. [19] report abnormal adenosine and dopamine receptor expression in enzyme deficient cells that are characteristic of Lesch-Nyhan syndrome, suggesting this might play a role in the neurological abnormality seen in the syndrome. Oliver et al. [20] and Sloneem et al. [21] report on a second syndrome initially thought to be closely associated with self-injury. In related studies they demonstrate that clinically significant self-injury in Cornelia de Lange syndrome (CdLS) is common (56\%) but at levels comparable to those seen in a matched contrast group. They also show that environmental influences on self-injury are as common in CdLS as the contrast group. Finally, Wulffaert et al. [22] report high levels of parental stress in families with a child with CHARGE syndrome, in which self-injury is commonly observed. This broader geneenvironment interaction warrants further attention.

\section{Behavioural Assessment}

Research within the applied behavioural field continues to grow with increasing focus placed upon modifications to functional analyses procedures in order to optimise outcome. Call, Pabico and Lomas [23] and Roscoe, Rooker, Pence and Longworth [24] demonstrated the utility of manipulating task aversivness in order to reveal a demand escape function to selfinjurious behaviour. These techniques borrow from the principles used in preference assessments by monitoring participant responses to an increasingly aversive hierarchy of demands. Additional antecedent manipulations are examined by Kuhn, Hardesty and Luczynski [25], who demonstrated that simple alterations in experimenter behaviour during tangible and attention conditions can result in differentiated functional analysis results without changing initial states of satiation or deprivation. These manipulations are also demonstrated by Tiger and colleagues [26**] who presented three case examples progressing from initially undifferentiated functional analysis assessments through to identification of idiosyncratic behavioural function. This study is particularly notable, as in addition to demonstrating proof of principle, the idiosyncratic behavioural function is confirmed through treatment evaluation. Interestingly, the authors note an association between one participant's diagnostic characteristics (autism spectrum disorder) and their idiosyncratic behavioural function of social escape. Knowledge of the characteristics associated with a particular neurodevelopmental disorder may help to focus attempts to uncover idiosyncratic functions to self-injurious behaviour. This highlights the need for synthesis between the applied behavioural and, for example, the behavioural phenotype literature. As assessment is further tailored to participant specific characteristics, research should seek to draw upon behavioural phenotype findings to direct hypotheses of potential idiosyncratic functions. 
Research has also focused upon minimising the relative risk and time involved in extended experimental functional analysis. Camp, Iwata, Hammond and Bloom [27] improved the utility of descriptive assessments by analysing conditional probabilities of both antecedent and consequence events. However, this still only produced correspondence with experimental functional analysis for four in seven participants. Herscovitch et al. [28*] provided an alternative solution to minimise risk of severe self-injury in experimental functional analysis. They described a procedure for identifying reliable precursors to self-injurious behaviour and then demonstrated that these precursors have the same behavioural function as the selfinjurious behaviour. This methodology has potential for clinical use and therefore the findings should be replicated and extended in a larger and more diverse sample.

A final noteworthy paper extends experimental functional analysis from humans to nonhuman primates [29*]. This research revealed that the self-injurious behaviour of a captive olive baboon had a social function that was identified using very similar experimental functional analysis methodologies as those used in humans.

\section{Psychological and pharmacological intervention}

Harvey et al. [30**] have updated a previously published meta-analysis of behavioural interventions for all challenging behaviour and applied different algorithms to evaluate effect size. They conclude that the evidence for behavioural interventions remains robust and emphasise the need for a functional analysis. The applied behaviour analytic literature continues to expand by demonstration of refinements to existing techniques. Davis et al. [31], Volkert et al. [32], Winborn-Kemmerer et al. [33] each demonstrate the value of functional communication training and the importance of the type of response that is selected, presession variables and schedules of reinforcement. Machalicek et al. [34] evaluated the use of activity schedules and task correspondence training with positive effects and Kerth et al. [35] modified self-restraint to good effect. Finally, a meta-analysis by Carr et al. [36*] of noncontingent reinforcement procedures concluded that there is robust evidence for the use of fixed time reinforcer delivery with extinction and schedule thinning. In combination these studies continue to draw attention to the very large and robust single case experimental design literature that shows behavioural approaches to be effective.

The context for this type of intervention is examined in studies by Lambrechts and Maes [37] and Lambrechts et al. [38] who report on the association between staff attributions, type of challenging behaviour and emotional reactions, and both staff responses to challenging behaviour and their estimates of the frequency of challenging behaviour. Understanding these relationships is important for the effectiveness of behavioural interventions. Finally, Petty et al. [39*] report on the observed temporal association between proto-self-injury, pragmatic communicative behaviours, repetitive behaviours and self-injury in young children with severe intellectual disability. This study provides tangential evidence for the emergence of self-injury from repetitive behaviours and the mechanism of operant social reinforcement.

Two studies report the results of controlled trials of medication. Marcus et al. [40] evaluated the efficacy of aripiprazole in children and adolescents with autism spectrum disorder and report a positive outcome but with two serious adverse events. Similarly Stigler et al. [41] evaluated the same intervention in pervasive developmental disorder not otherwise specified and Asperger's disorder reporting $88 \%$ of 25 participants to be 'responders' with no adverse events reported. In both cases a broad assessment of 'irritability' was used and thus specific effects on self-injury are unknown. Aman et al. [42**] report that risperidone combined with 
parent training was superior to risperidone alone in improving severe behavioural problems, including self-injury. The latter study indicates the need for research and intervention to acknowledge both biological and environmental influences and the studies of aripiprazole indicate a clear need for more focused outcome measures.

\section{Conclusion}

The prevalence and cohort description literature continues to be refined and integrated into models of the causes of self-injury and this is a welcome development that will help develop more complete accounts. The studies of self-injury in groups defined by aetiology allude to interactions between individual characteristics and the environment that will help refine and improve intervention if robust causal models being to emerge and there is good evidence that this is the case. The studies of Aman et al. [42**], Symons et al. [18**] and May et al. $\left[17^{* *}\right]$ open new avenues of research that are promising and warrant attention and resources. Finally, the evidence for behavioural interventions continues to grow but the emphasis should perhaps shift from demonstration to delivery. 


\section{Acknowledgements}

The authors are generously supported by grants from Cerebra (CO) and Research Autism (CR). Hayley Mace provided invaluable support to the authors. 


\section{References and recommended reading}

Papers of particular interest published within the annual period of review have been highlighted as:

* of special interest

** of outstanding interest

1 ** Cooper SA, Smiley E, Allan LM, et al. Adults with intellectual disabilities: prevalence, incidence and remission of self-injurious behaviour, and related factors. Journal of Intellectual Disability Research. 2009 Mar;53(3):200-16.

This was a well conducted large scale study, longitudinally examining the prevalence, incidence and remission rates of self-injurious behaviour.

2 Cohen IL, Tsiouris JA, Flory MJ, et al. Large scale study of the psychometric characteristics of the IBR modified overt aggression scale: findings and evidence for increased selfdestructive behaviors in adult females with autism spectrum disorder. J Autism Dev Disord. 2010 May;40(5):599-609.

3 Murphy O, Healy O, Leader G. Risk factors for challenging behaviors among 157 children with autism spectrum disorder in Ireland. Research in Autism Spectrum Disorders. 2009 AprJun;3(2):474-82.

4 Danquah A, Limb K, Chapman M, et al. An investigation of factors predictive of continued self-injurious behaviour in an intellectual disability service. Journal of Applied Research in Intellectual Disabilities. 2009 Jul;22(4):395-9.

5 Matson JL, Boisjoli J, Mahan S. The relation of communication and challenging behaviors in infants and toddlers with autism spectrum disorders. Journal of Developmental and Physical Disabilities. 2009 Aug;21(4):253-61.

6 Matson JL, Shoemaker M. Intellectual disability and its relationship to autism spectrum disorders. Research in Developmental Disabilities. 2009 Nov-Dec;30(6):1107-14.

$7 \quad$ Felce D, Kerr M, Hastings RP. A general practice-based study of the relationship between indicators of mental illness and challenging behaviour among adults with intellectual disabilities. Journal of Intellectual Disability Research. 2009 Mar;53(3):243-54.

8 McCarthy J, Hemmings C, Kravariti E, et al. Challenging behavior and co-morbid psychopathology in adults with intellectual disability and autism spectrum disorders. Research in Developmental Disabilities. 2010 Mar-Apr;31(2):362-6.

$9 * * \quad$ Holden B, Gitlesen JP. The overlap between psychiatric symptoms and challenging behaviour: A preliminary study. Research in Developmental Disabilities. 2009 MarApr;30(2):210-8.

An important study investigating the overlap between psychiatric symptoms and challenging behaviour providing useful empirical evidence for an increased distinction between the two.

10 Brown J, Beail N. Self-harm among people with intellectual disabilities living in secure service provision: a qualitative exploration. Journal of Applied Research in Intellectual Disabilities. 2009 Nov;22(6):503-13.

11 Byrne A, Hennessy E. Understanding challenging behaviour: perspectives of children and adolescents with a moderate intellectual disability. Journal of Applied Research in Intellectual Disabilities. 2009 Jul;22(4):317-25.

12 ** Love JR, Carr JE, LeBlanc LA. Functional assessment of problem behavior in children with 
autism spectrum disorders: A summary of 32 outpatient cases. J Autism Dev Disord. 2009 Feb;39(2):363-72.

This study usefully begins to delineate function of self-injurious behaviour in individuals with autism spectrum disorder using a variety of experimental methodologies.

13* O'Reilly M, Rispoli M, Davis T, et al. Functional analysis of challenging behavior in children with autism spectrum disorders: A summary of 10 cases. Research in Autism Spectrum Disorders. 2010 Jan-Mar;4(10):1-10.

A very interesting study reporting a novel finding of predominantly automatic reinforcement for challenging behaviour in individuals with autism spectrum disorder.

14 Lundqvist L, Andersson G, Viding J. Effects of vibroacoustic music on challenging behaviors in individuals with autism and developmental disabilities. Research in Autism Spectrum Disorders. 2009 Feb;3(2): 390-400.

15* Soares DA, Vannest KJ, Harrison J. Computer aided self-monitoring to increase academic production and reduce self-injurious behaviour in a child with Autism. Behavioral Interventions. 2009 Apr;24(3):171-183.

An interesting and well controlled single case study.

16* Tiger JH, Fisher WW, Bouxsein KJ. Therapist- and self-monitored DRO contingencies as a treatment for the self-injurious skin picking of a young man with Asperger syndrome. J Appl Behav Anal. 2009 Summer;42(2):315-9.

A well described experimental case study.

17** May ME, Srour A, Hedges LK, et al. Monoamine oxidase a promoter gene associated with problem behavior in adults with intellectual/ developmental disabilities. American Association on Intellectual and Developmental Disabilities. 2009 Jul;114(4):269-73. Potentially a very important study demonstrating an association between challenging behaviour and genetic variability not related to a specific syndrome of genetic origin.

18** Symons FJ, Harper VN, McGrath PJ et al. Evidence of increased non-verbal behavioral signs of pain in adults with neurodevelopmental disorders and chronic self-injury. Research in Developmental Disabilities, 2009 May-Jun;30(3):521-528.

Pain is frequently overlooked as an important variable in the study and treatment of self-injury. An important study that demonstrates the application of useful observational methodology.

19 García MG, Puig JG, Torres RJ. Abnormal adenosine and dopamine receptor expression in lymphocytes of Lesch-Nyhan patients. Brain Behav Immun. 2009 Nov;23(8):1125-31.

20 Oliver C, Sloneem J, Hall S, Arron K. Self-injurious behaviour in Cornelia de Lange syndrome: 1. Prevalence and phenomenology. Journal of Intellectual Disability Research. 2009 Jul;53(7):575-89.

21 Sloneem J, Arron K, Hall S, Oliver C. Self-injurious behaviour in Cornelia de Lange syndrome: 2. association with environmental events. Journal of Intellectual Disability Research. 2009 Jul;53(7):590-603.

22 Wulffaert J, Scholte EM, Dijkxhoorn YM, et al. Parenting stress in CHARGE Syndrome and the relationship with child characteristics. Journal of Developmental and Physical Disabilities, 2009 Aug;21(4):301- 13.

23 Call NA, Pabico RS, Lomas JA. Use of latency to problem behavior to evaluate demands for inclusion in functional analyses. J Appl Behav Anal. 2009 Fall;42(3):723-8 
24 Roscoe EM, Rooker GW, Pence ST, Longworth LJ. Assessing the utility of a demand assessment for functional analysis. J Appl Behav Anal. 2009 Winter;42(4):819-25.

25 Kuhn DE, Hardest SL, Luczynski K. Further evaluation of antecedent social events during functional analysis. J Appl Behav Anal. 2009 Summer;42(2):349-53.

26** Tiger JH, Fisher WW, Toussaint KA, Kodak T. Progressing from initially ambiguous functional analyses: Three case examples. Research in Developmental Disabilities. 2009 SepOct;30(5):910-26.

A very useful study detailing results from idiosyncratic manipulations to experimental functional analysis.

27 Camp EM, Iwata BA, Hammond JL, Bloom SE. Antecedent versus consequent events as predictors of problem behavior. J Appl Behav Anal. 2009 Summer;42(2):469-83.

28* Herscovitch B, Roscoe EM, Libby ME, et al. A procedure for identifying precursors to problem behavior. Journal of Applied Behavior Analysis. 2009 Fall;42(3), 697-702.

An interesting study providing a robust methodology for identifying precursor behaviours.

29* Dorey NR., Rosales-Ruiz J, Smith R, Lovelace B. Functional analysis and treatment of selfinjury in a captive olive baboon. J Appl Behav Anal. 2009 Winter;42(4):785-94.

A novel and interesting application of experimental functional analysis.

30** Harvey ST, Boer D, Meyer LH, Evans IM. Updating a meta-analysis of intervention research with challenging behaviour: Treatment validity and standards of practice. Journal of Intellectual \& Developmental Disability. 2009;34(1):67-80.

An important update of a prior meta-analysis with robust and systematic evaluation of effect sizes.

31 Davis TN, O'Reilly MF, Kang S, et al. Impact of presession access to toys maintaining challenging behavior on functional communication training: A single case study. Journal of Developmental and Physical Disabilities. 2009 Dec; 21(6):515- 21.

32 Volkert VM, Lerman DC, Call NA, Trosclair-Lasserre N. An evaluation of resurgence during treatment with functional communication training. J Appl Behav Anal. 2009 Spring; 42(1):145-60.

33 Winborn-Kemmerer L, Ringdahl JE, Wacker DP, Kitsukawa K. A demonstration of individual preference for novel mands during functional communication training. J Appl Behav Anal. 2009 Spring;42(1):185-189.

34 Machalicek W, Shogren K, Lang R, et al. Increasing play and decreasing the challenging behavior of children with autism during recess with activity schedules and task correspondence training. Research in Autism Spectrum Disorders. 2009 Apr-Jun;3(2):547-55.

35 Kerth DM, Progar PR., Morales S. The effects of non-contingent self-restraint on self-injury. Journal of Applied Research in Intellectual Disabilities. 2009 Mar;22(2):187-93.

36* Carr JE, Severtson JM, Lepper TL. Noncontingent reinforcement is an empirically supported treatment for problem behavior exhibited by individuals with developmental disabilities, Research in Developmental Disabilities. 2009 Summer;30(1):469-83.

Meta-analytic approach to evaluate one form of behavioural intervention with robust evidence for efficacy. 
37 Lambrechts G, Maes B. Analysis of staff reports on the frequency of challenging behaviour in people with severe or profound intellectual disabilities. Research in Developmental Disabilities. 2009 Sep-Oct;30(5):863-72.

38 Lambrechts G, Kuppens S, Maes B. Staff variables associated with the challenging behaviour of clients with severe or profound intellectual disabilities. Journal of Intellectual Disability Research. 2009 Jul;53(7):620-32.

39* Petty J, Allen D, Oliver C. Relationship among challenging, repetitive, and communicative behaviors in children with severe intellectual disabilities. American Association on Intellectual and Developmental Disabilities. 2009 Sep;114(5):356-68.

Of interest because of the implications for early intervention.

40 Marcus RN, Owen R, Kamen L, et al. A placebo-controlled, fixed-dose study of aripiprazole in children and adolescents with irritability associated with autistic disorder. J Am Acad Child Adolesc Psychiatry. 2009 Nov;48(11):1110-9.

41 Stigler KA, Diener JT, Kohn AE, et al. Aripiprazole in pervasive developmental disorder not otherwise specified and Asperger's disorder: a 14-week, prospective, open-label study. J Child Adolesc Psychopharmacol. 2009 Jun;19(3):265-74.

42** Aman MG, McDougle CJ, Scahill L, et al. Medication and parent training in children with pervasive developmental disorders and serious behavior problems: Results from a randomized clinical trial. J Am Acad Child Adolesc Psychiatry. 2009 Dec;48(12):1143-1154.

A robust experimental design to evaluate the combined efficacy of parent training and risperidone. Notable for the integration of different perspectives. 